OPEN ACCESS

Edited by:

Xi-Nian Zuo,

Beijing Normal University, China

Reviewed by:

Hewei Cheng,

Chongqing University of Posts and Telecommunications, China

Qinmu Peng,

Huazhong University of Science and Technology, China

${ }^{*}$ Correspondence:

Jinping $X u$

jp.xu@siat.ac.cn

Bochao Cheng

wonder9527@163.com

Jiaojian Wang

jiaojianwang@uestc.edu.cn

${ }^{\dagger}$ These authors have contributed equally to this work

Specialty section: This article was submitted to Brain Imaging Methods, a section of the journal Frontiers in Neuroscience

Received: 13 January 2020 Accepted: 07 April 2020

Published: 27 May 2020

Citation:

Gao J, Zeng $M$, Dai $X$, Yang $X$, Yu H, Chen K, Hu Q, Xu J, Cheng B

and Wang $J$ (2020) Functional Segregation of the Middle Temporal Visual Motion Area Revealed With

Coactivation-Based Parcellation.

Front. Neurosci. 14:427.

doi: 10.3389/fnins.2020.00427

\section{Functional Segregation of the Middle Temporal Visual Motion Area Revealed With Coactivation-Based Parcellation}

\author{
Jingjing Gao't, Min Zeng't, Xin Dai ${ }^{3}$, Xun Yang ${ }^{4}$, Haibo $\mathrm{Yu}^{5}$, Kai Chen ${ }^{6}$, Qingmao $\mathrm{Hu}^{7,8}$, \\ Jinping $\mathrm{Xu}^{8 *}$, Bochao Cheng ${ }^{9 *}$ and Jiaojian Wang ${ }^{10,11 *}$
}

${ }^{1}$ School of Information and Communication Engineering, University of Electronic Science and Technology of China, Chengdu, China, ${ }^{2}$ Department of Radiology, Pidu District People's Hospital, Chengdu, China, ${ }^{3}$ School of Automation, Chongqing University, Chongqing, China, ${ }^{4}$ School of Public Affairs, Chongqing University, Chongqing, China, ${ }^{5}$ Department of Acupuncture and Moxibustion, Shenzhen Traditional Chinese Medicine Hospital, Shenzhen, China, ${ }^{6}$ School of Automation Engineering, University of Electronic Science and Technology of China, Chengdu, China, ${ }^{7}$ Institute of Biomedical and Health Engineering, Shenzhen Institutes of Advanced Technology, Chinese Academy of Sciences, Shenzhen, China, ${ }^{8}$ CAS Key Laboratory of Human-Machine Intelligence-Synergy Systems, Shenzhen Institutes of Advanced Technology, Chinese Academy of Sciences, Shenzhen, China, ${ }^{9}$ Department of Radiology, West China Second University Hospital of Sichuan University, Chengdu, China, ${ }^{10}$ School of Life Sciences and Technology, University of Electronic Science and Technology of China, Chengdu, China, ${ }^{11}$ Center for Language and Brain, Shenzhen Institute of Neuroscience, Shenzhen, China

Traditionally, the visual motion area (MT) is considered as a brain region specialized for visual motion perception. However, accumulating evidence showed that MT is also related to various functions, suggesting that it is a complex functional area and different functional subregions might exist in this area. To delineate functional subregions of this area, left and right masks of MT were defined using meta-analysis in the BrainMap database, and coactivation-based parcellation was then performed on these two masks. Two dorsal subregions ( $\mathrm{Cl} 1$ and $\mathrm{Cl} 2)$ and one ventral subregion (Cl3) of left $\mathrm{MT}$, as well as two dorsal-anterior subregions ( $\mathrm{Cl} 1$ and $\mathrm{Cl} 2$ ), one ventral-anterior subregion (Cl3), and an additional posterior subregion ( $\mathrm{Cl} 4)$ of right MT were identified. In addition to vision motion, distinct and specific functions were identified in different subregions characterized by task-dependent functional connectivity mapping and forward/reverse inference on associated functions. These results not only were in accordance with the previous findings of a hemispheric asymmetry of MT, but also strongly confirmed the existence of subregions in this region with distinct and specific functions. Furthermore, our results extend the special role of visual motion perception on this area and might facilitate future cognitive study.

Keywords: MT, visual motion perception, meta-analysis, coactivation-based parcellation, meta-analytic connectivity mapping

\section{INTRODUCTION}

The human being acquires outer information mainly relying on visual inputs. Visual motion perception is primarily modulated by the visual motion area (MT), which is a brain region specialized for the perception of motion in the visual modality. The first discovery of MT comes from the study of stimulating sensitive area of visual movement in the monkey brain 
(Allman and Kaas, 1971; Dubner and Zeki, 1971; Zeki, 1974). Subsequently, a positron emission tomography (PET) study confirmed the existence of a homologous counterpart in human brain (Zeki et al., 1991). Although the MT is identified in human brain, the exact location of MT is still under debate. Using PET technology, Zhang et al. (2007) studied 12 normal movement and static visual tasks and consistently found that the MT area was located in the occipital lobe in spite of individual variability. This area was exactly located in the junction between the ascending limb of the inferior temporal sulcus and the lateral occipital sulcus (Watson et al., 1993). Using functional magnetic resonance imaging (fMRI), Dumoulin et al. (2000) found that the location of MT mainly included three sulcus: the inferior temporal sulcus (11\%), temporal sulcus rising branch (53\%), and the posterior continuations of inferior temporal sulcus (46\%). MT was recently further delineated by analysis of myelination, and MT has significantly different myelination compared to surrounding tissues (Annese et al., 2004).

The traditional view of MT is that this area mainly responds to visual motion. A large number of recent studies have demonstrated that this area is also related to motion of auditory and tactile (Howard et al., 1995; Poirier et al., 2006; Ricciardi et al., 2007; Watkins et al., 2013; Abdollahi et al., 2014). Different ways of stimulation result in different response areas of MT (Morrone et al., 2000). Using fMRI, Smith et al. (2006) further demonstrated that there are different functional subregions in MT: the lateral MT was significantly activated by the optic flow stimuli from the contralateral side, whereas the other subregion, medially MST, was significant activated by the optic flow stimuli from the same side (Smith et al., 2006). Moreover, MT was reported to be involved into spatial deep perception, shape detection, and binocular rivalry (Ferri et al., 2013). All these evidence suggested that MT is a complex functional area and different functional subregions exist in this area.

More and more studies have demonstrated that the brain functions were determined by its different connectivity patterns with other brain areas (Wang et al., 2012, 2015a; Xu et al., 2015, 2019a). Using anatomical and resting-state functional connectivity-based parcellation approach, many brain areas have been subdivided into different functional subregions (Wang et al., 2015b, 2016; Xu et al., 2019b). Recently, Eickhoff et al. (2011) proposed task-related coactivation-based parcellation approach to parcellate the brain with BrainMap database ${ }^{1}$ to characterize the functional organization of the brain under task. Coactivation-based parcellation results showed similarities with the findings derived from anatomical and resting-state functional connectivity-based parcellation (Wang et al., 2015b, 2017, 2019). Moreover, given coactivation-based parcellation characterizing the task-related connectivity, it is thus better to investigate the brain functional areas that do not have clear anatomical boundary.

In our study, we first defined left and right functional masks of MT using meta-analysis in the BrainMap database. Then, we performed coactivation-based parcellation of left and right MT to

${ }^{1}$ http://www.brainmap.org/ identify functional subregions. Finally, we characterized the taskrelated connectivity and functions for each subregion using metaanalytic connectivity mapping (MACM).

\section{MATERIALS AND METHODS}

\section{Definition of MT Area Masks}

Motion area is specific for visual motion and approximately located in the junction of the posterior middle temporal gyrus (MTG) and occipital gyrus (Smith et al., 2006). Given no consensus of neuroanatomical landmarks to define the location of MT, thus, we used meta-analysis of visual motion task in BrainMap database to identify MT. After obtaining the coordinates of the experiments for visual motion, activation likelihood estimation (ALE) was applied to model the functional activation, and $p<0.001$ with false discovery rate (FDR) correction was used to identify the functional activations to define the functional masks for MT for parcellation.

\section{Coactivation-Based Parcellation}

Coactivation-based parcellation approach was used to identify the functional subregions of MT in this study. The whole-brain coactivation connectivity calculated using MACM was further used to define the coactivation profile for each MT voxel with BrainMap database (Laird et al., 2009, 2011). Only PET and fMRI experiments reporting stereotaxic coordinates in healthy subjects were entered into our analyses. In BrainMap database, the tasks ranged from executive functions to sensorimotor processing and cognition, such as inhibition control, working memory, and language processing. Voxels in the neighborhood of each seed voxel in MT were pooled and used to define the task-related coactivation pattern. By computing and sorting the Euclidian distances between a given seed voxel and any reported activation coordinate, those experiments reporting activation coordinates closest to the present seed voxel were identified with the extent of the spatial filter from 20 to 200 experiments in steps of 5. Next, an ALE meta-analysis of the experiments associated with that particular voxel was performed, and the ALE scores were taken as the whole-brain coactivation pattern (Eickhoff et al., 2009, 2012; Turkeltaub et al., 2012; Bzdok et al., 2013; Cieslik et al., 2013). The coactivation patterns for all the seed voxels were combined into an $N \times M$ matrix, where $N$ is the number of seed voxels in the MT and $M$ is the number of whole-brain voxels. The similarity was defined using one minus the correlation between each pair of the coactivation patterns of the seed voxels in MT. Finally, MT parcellation was performed using $\mathrm{K}$-means clustering method with $K=2$, 3, .., 7 in the optimal filter (Clos et al., 2013). The optimal filter range was determined by assessing the consistency of the cluster assignments for individual voxels across different filter sizes, and the range with the lowest number of deviants was selected as the optimize.

\section{Determination of the Number of Clusters}

The index of hierarchically inconsistent voxels was used to determine the optimal number of clusters of the MT. The index 
of hierarchically inconsistent voxels quantifies the percentage of voxels not related to the dominant parent cluster compared to the K-1 clustering number. The lowest lost voxel was considered as the optimal clustering solution (Kahnt et al., 2012; Clos et al., 2013).

\section{Whole-Brain Coactivation Patterns of Each Subregion}

Structure-based meta-analysis was used to map the taskbased coactivation patterns for each subregion of MT in the BrainMap database. To map the coactivation pattern, at least one focus of activation in a particular subregion was pooled (Eickhoff et al., 2010; Robinson et al., 2010; Laird et al., 2013). An ALE meta-analysis on the experiments and statistical inference were performed to identify brain regions that significantly coactivated with a particular subregion. Then, the ALE score was compared to a null-distribution to determine the above-chance convergence between experiments (Eickhoff et al., 2009). The ALE scores activated within a particular subregion were tested against the ALE scores obtained under this null-distribution, yielding a $p$-value on the basis of the proportion of equal or higher random values (Eickhoff et al., 2012). These non-parametric p-values were finally converted to $z$-scores and thresholded at $p<0.05$ (cluster-level FWE-corrected, cluster-forming threshold at voxellevel, $p<0.001)$.

\section{Specific Coactivation Pattern for Each Subregion}

The specific coactivation pattern was calculated to identify the unique task-related coactivation patterns for each MT subregion compared to other subregions. The specific coactivation patterns were the brain areas that were significantly more coupled with a given subregion than that with any of the others.

\section{Functional Characterization of Each Subregion}

Each MT subregion was functionally characterized based on behavioral domain and paradigm class using the BrainMap database. Functional characterization of each MT subregion was determined using forward and reverse inferences (Bzdok et al., 2013; Cieslik et al., 2013; Clos et al., 2013; Rottschy et al., 2013; Wang et al., 2015b). In the forward inference approach, the functional profile of a specific subregion was determined by identifying the domains or subdomains for which the probability of activation was significantly higher than the overall chance of activation in that particular subregion. Significance was established using a binomial test $(p<0.05$ corrected for multiple comparisons using FDR method) (Eickhoff et al., 2011). In the reverse inference approach, the functional profile of a subregion was determined by identifying the most likely behavioral domains and paradigm classes associated with activation in a particular subregion. Significance was then assessed by means of a Chi-squared test $(p<0.05$ corrected for multiple comparisons using Bonferroni's method) (Clos et al., 2013).

\section{RESULTS}

\section{Coactivation-Based Parcellation Result}

The locations of human left and right MT were defined (Figure 1A). Moreover, the left and right MT areas were parcellated into different clusters ranging from 2 to 7 at the optimal filter size of $115-165$ and $100-145$, respectively. The hierarchical inconsistency index-identified optimal parcellation schemes for left and right MT were three and four subregions, respectively (Figure 1B). The three- and four-way parcellation of the left and right MT were used to guide the following analyses. In the left MT area, three subregions were identified (Figure 1C). Two subregions were located in the dorsal $\mathrm{MT}(\mathrm{Cl} 1$ and $\mathrm{Cl} 2)$, and one subregion was located in the ventral MT $(\mathrm{Cl} 3)$. The right MT was parcellated into four subregions (Figure 1C). Two subregions were located in the dorsal-anterior MT (Cl1 and $\mathrm{Cl} 2)$, and one subregion was located in the ventral-anterior MT $(\mathrm{Cl} 3)$. Moreover, an additional posterior subregion $(\mathrm{Cl} 4)$ in the right MT was also identified.

\section{Coactivation Pattern of Each MT Subregion}

To uncover the task-related connectivity pattern for each MT subregion, MACM for each subregion was performed, and the coactivation pattern for each subregion was shown in Figure 2. For the left $\mathrm{Cl1}$, the coactivated brain areas were bilateral precentral gyrus (PCG), rostral supramarginal gyrus (SMG), angular gyrus (AG), supplementary motor area (SMA), intraparietal sulcus (IPS), inferior/middle/superior occipital gyrus (I/M/SOG), left inferior frontal gyrus (IFG), and the right posterior superior temporal gyrus (STG). For the left $\mathrm{Cl} 2$, the coactivated brain areas were found in bilateral PCG, SMA, AG, IPS, I/M/SOG, left IFG, rostral SMG, right STG, and IFG. The left $\mathrm{Cl} 3$ primarily coactivated with bilateral PCG, IFG, SMA, AG, IPS, $\mathrm{I} / \mathrm{M} / \mathrm{SOG}$, and right middle frontal gyrus (MFG). For the right $\mathrm{Cl} 1$, the coactivated brain areas were bilateral IPS, AG, I/M/SOG, SMA, and right PCG. The right $\mathrm{Cl} 2$ mainly coactivated with bilateral PCG, SMA, rostral SMG, IPS, I/M/SOG, right STG, and left IFG. The right $\mathrm{Cl} 3$ coactivated with bilateral PCG, IPS, AG, SMA, and I/M/SOG. For the right $\mathrm{Cl} 4$, the main coactivation was observed in bilateral PCG, IFG, SMA, IPS, AG, and I/M/SOG.

\section{Specific Coactivation Pattern of Each MT Subregion}

The specific task-related connectivity for each MT subregion was mapped to identify the unique coactivation pattern. For the left $\mathrm{Cl1}$, the specific coactivation was found in left IFG, IPS, rostral SMG, right SMG, and posterior MTG (Figure 3). For the left $\mathrm{Cl} 2$, the unique task-dependent connectivity was observed in bilateral IPS, AG, and right posterior MTG (Figure 3). The left $\mathrm{Cl} 3$ specifically coactivated with bilateral PCG, SMA, IFG, and right SMG (Figure 3). The right $\mathrm{Cl}$ 1 specifically coactivated with left posterior MTG (Figure 3). For the right $\mathrm{Cl} 2$, the unique coactivation was found in left IFG, MT, and right PCG (Figure 3). The specific connection under task for right $\mathrm{Cl} 3$ was found in left rostral SMG, posterior MTG, and right PCG, AG (Figure 3). The 


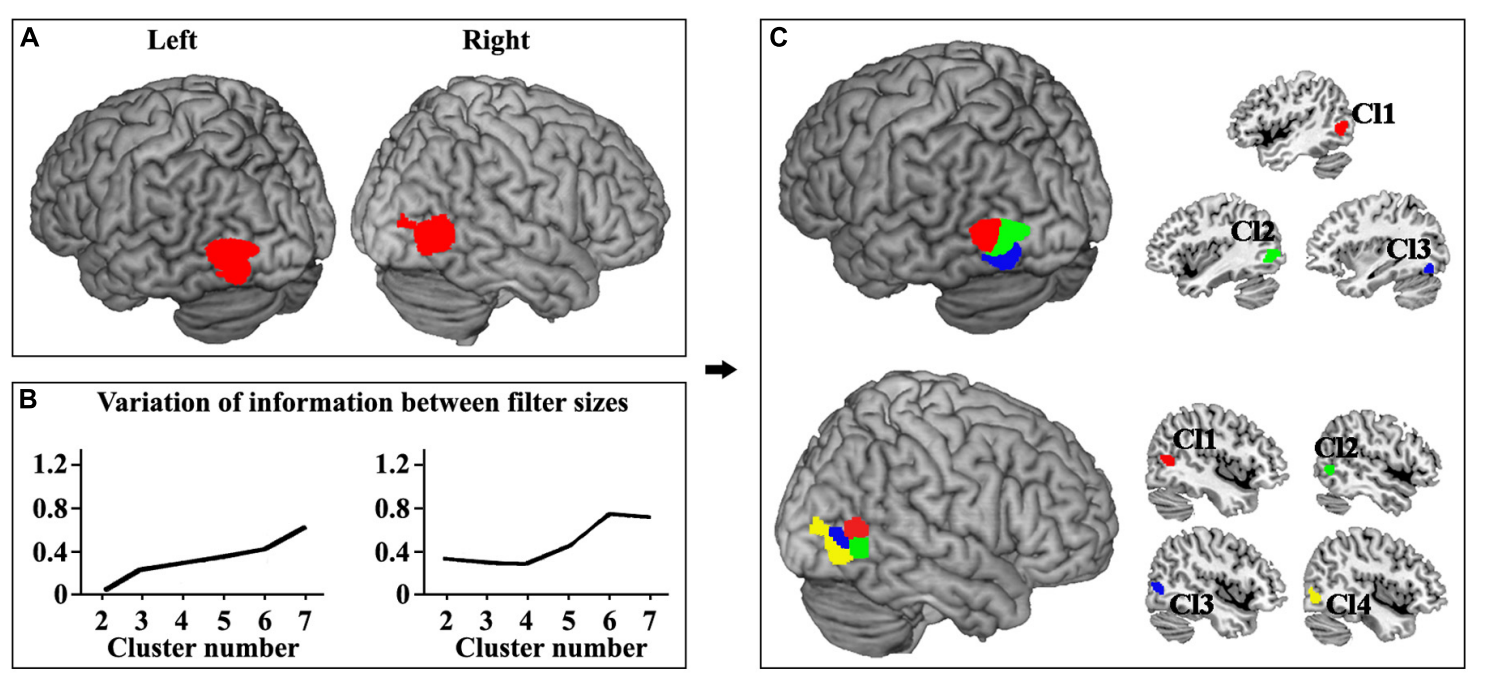

FIGURE 1 | (A) The locations of human left and right MT were defined using meta-analysis of visual motion task in BrainMap database. The false discovery rate (FDR) correction with $p<0.001$ was used. (B) The left and right MT areas were parcellated into different clusters ranging from 2 to 7 at the optimal filter size of 115-165 and 100-145, respectively. The hierarchical inconsistency index-identified optimal parcellation schemes for left and right MT were three and four subregions, respectively. (C) The three-way parcellation of the left $\mathrm{MT}(\mathrm{Cl} 1, \mathrm{Cl} 2$, and $\mathrm{Cl} 3)$ and four-way parcellation of the right $\mathrm{MT}(\mathrm{Cl}$, $\mathrm{Cl} 2$, $\mathrm{Cl} 3$, and $\mathrm{Cl} 4)$ were shown and used to guide the following analyses.

A

Cl1

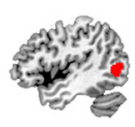

$\mathrm{Cl2}$

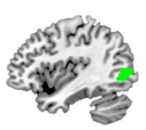

$\mathrm{Cl3}$

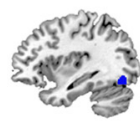

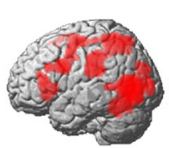
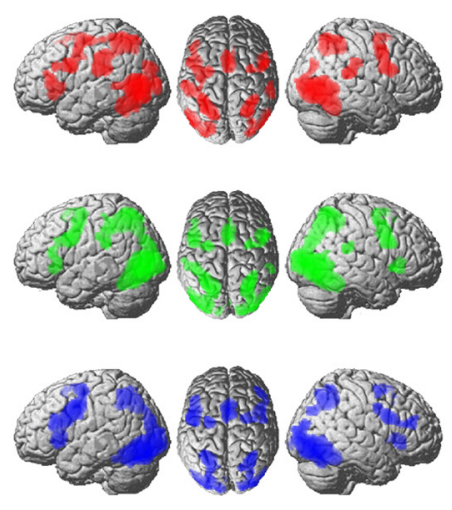

B

Cl1

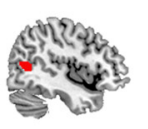

$\mathrm{Cl2}$

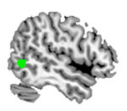

Cl3
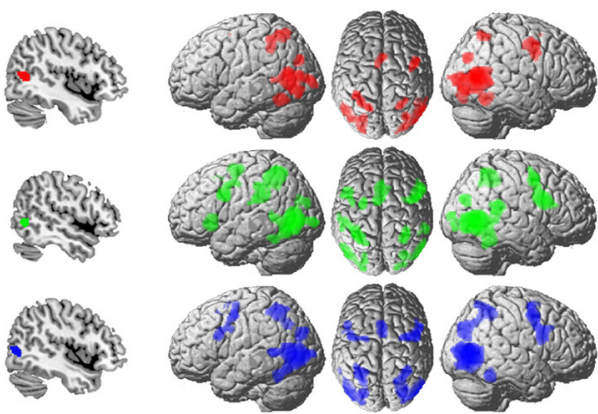

Cl4
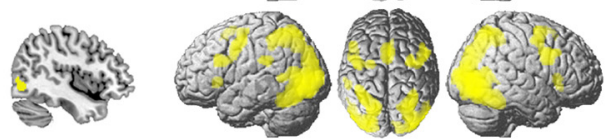

FIGURE 2 | The whole-brain coactivation pattern for each subregion of left (A) and right (B) MT was obtained using meta-analytical connectivity modeling analyses. The significance levels were set at $p<0.05$, cluster-level FWE-corrected, and cluster-forming threshold at voxel-level $p<0.001$.

right Cl4 specifically coactivated with left IPS, posterior MTG, and right SMG (Figure 3).

\section{Functional Characterization}

Quantitative functional characterization of each MT subregion was also performed (Figure 4). For the left $\mathrm{Cl1}$, the main functions associated with this area were action observation and sexuality. The Cl1 was also related to visual shape and motion perception. For the left $\mathrm{Cl} 2$, the main functions of this area were sexuality, action observation, and visual shape perception. The left $\mathrm{Cl} 2$ was also associated with visual motion perception, space cognition, and attention. The left $\mathrm{Cl} 3$ was mainly associated with visual motion and shape perception and semantic processing. For the right $\mathrm{Cl}$, the main function was visual motion perception.
The main function for the right $\mathrm{Cl} 2$ was action observation. The functions of visual motion and shape perception for the right $\mathrm{Cl} 2$ were also found. The right $\mathrm{Cl} 3$ mainly participated in soma, visual motion, and action observation. The functions of visual perception, spatial cognition, and sexuality for the right $\mathrm{Cl} 3$ were also found. The right $\mathrm{Cl} 4$ primarily participated in activation observation, soma, spatial cognition, visual shape and motion perception, and sexuality. From the coactivation pattern and functional characterization for each subregion, we found that the left subregion of $\mathrm{Cll}$ (red one) mainly corresponds to the right $\mathrm{Cl} 2$ subregion (green one); the left subregion of $\mathrm{Cl} 2$ (green one) mainly corresponds to the right $\mathrm{Cl} 3$ subregion (blue one); and the left subregion of $\mathrm{Cl} 3$ (blue one) mainly resembles the right $\mathrm{Cl} 4$ subregion (yellow one). Furthermore, we identified a specific 


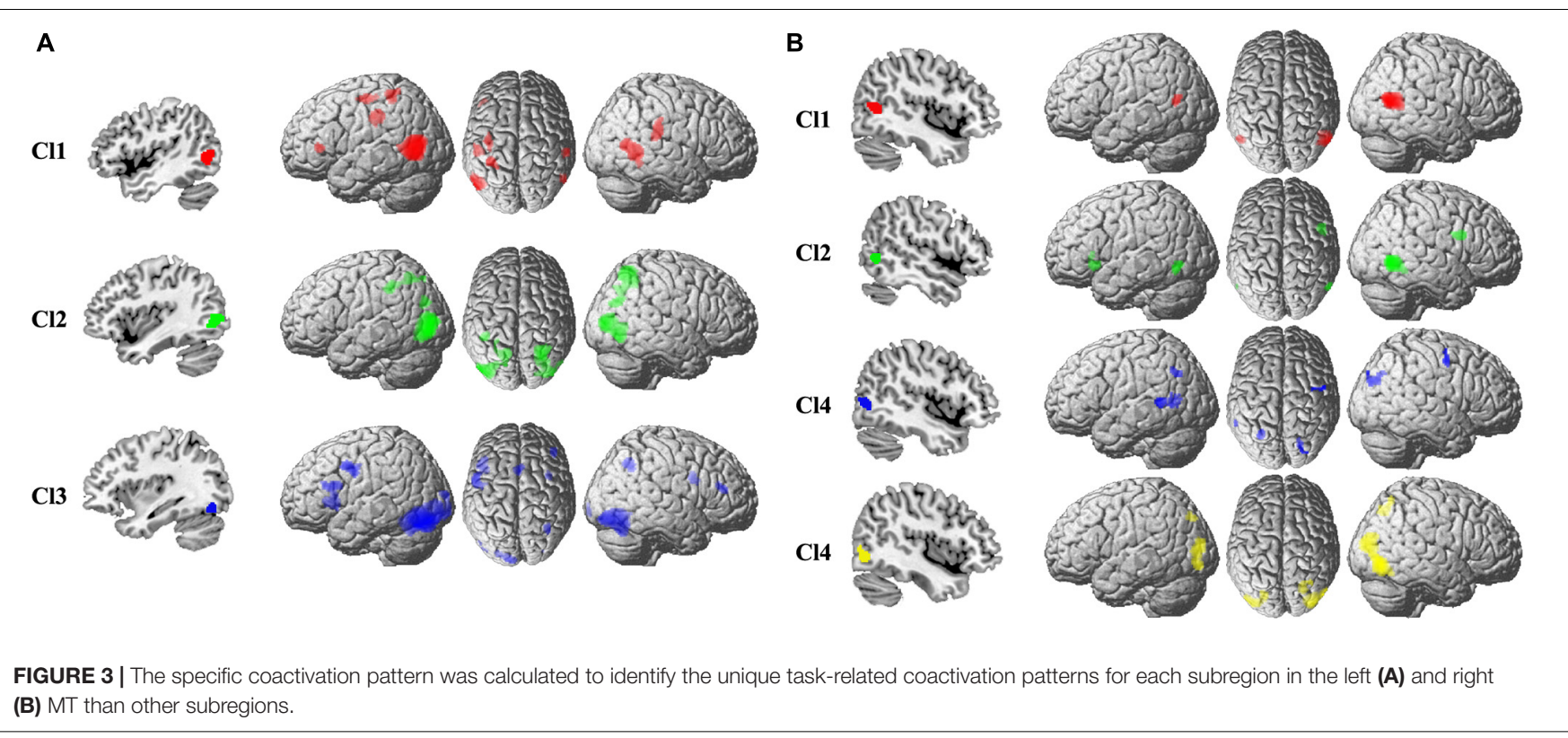

subregion of right $\mathrm{Cl} 1$ that is mainly responsible for visuospatial attention processing.

\section{DISCUSSION}

In this study, we proposed a parcellation scheme for the bilateral MT based on whole-brain MACM in the BrainMap database. Three distinct subregions of left MT and four distinct subregions of right MT were identified. In addition to vision motion, distinct and specific functions were identified in different subregions characterized by task-dependent functional connectivity mapping and forward/reverse inference on associated functions.

Notably, multi-functional characteristics of human MT were put forward, supporting that it is not a single area but a complex of areas with several distinct functional subareas. Some of these subareas are likely to be the homologues of monkey MT and its satellites MST and FST (Ferri et al., 2013). To date, many methods were widely performed to reveal the structural and functional definitions of human MT in vivo, including motion localizer test (Zeki et al., 1991; Tootell et al., 1995), employing patterns of myelination (Glasser and van Essen, 2011; Bridge et al., 2014), retinotopic mapping techniques (Huk et al., 2002; Kolster et al., 2010), and quantitative T1 mapping (Sereno et al., 2013). The MT was first subdivided into two distinct areas in the monkey brain, one is MT and the other is MST (Huk et al., 2002). Based on more exact measurement of retinotopic map of MT, previous studies have demonstrated at least two retinotopically mapped regions including TO1 and TO2, which correspond to the MT and MST in the monkey brain, respectively (Amano et al., 2009; Kolster et al., 2010). Subsequently, Kolster et al. (2010) segment the motion-sensitive MT cluster into four subregions, respectively, referred to as MT proper, putative MSTv (putative ventral part of the medial superior temporal area), putative FST (fundus of the superior temporal area), and putative V4t (transitional zone) by combining functional MRI and the retinotopic mapping technique. This retinotopic organization in humans was very similar to that documented in the monkey. Shortly after, Glasser and van Essen (2011) used an automated approach to delineate cortical areas based on myelin gradients computed from the ratio of T1- and T2-weighted images collected at $3 \mathrm{~T}$ in vivo. They identified a large area of strong myelination as the MT complex comprising a number of constituent areas including putative hoc5. However, two previous studies (Abdollahi et al., 2014; Bridge et al., 2014) have compared the definition results of human MT employing patterns of myelination and retinotopic mapping techniques using two complementary approaches. They found that the total number of myelin content within the cortical ribbon was significantly increased. Moreover, Sereno et al. (2013) found that the region of dense myelination on the lateral occipital surface was considerably larger than retinotopically defined MT by comparing quantitative T1 mapping with the retinotopic mapping technique. These results strongly suggested that different studies using different methods resulted in different segmentation of MT regions. Moreover, more challenging is on the way to investigate human myelo-architecture patterns in vivo in the human extrastriate regions for individual subjects (Sanchez-Panchuelo et al., 2012). Given these discrepancies and challenging, complementary methods just as we used may provide more additional distinction to reveal the structural and functional definition and parcellation of human MT. Indeed, using meta-analysis of visual motion task in BrainMap database, the locations of human bilateral MT were defined, and they were further subdivided into three distinct subregions in the left MT and four distinct subregions in the right MT based on coactivation-based parcellation in the current study. These results not only were in accordance with the previous findings of a hemispheric asymmetry of MT (Ohlendorf et al., 2008; Kolster et al., 2010), but also strongly confirmed the existence of subregions in this region (Kolster et al., 2010; Bridge et al., 2014). 
A

Cl1
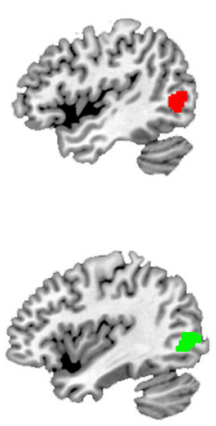

Cl3

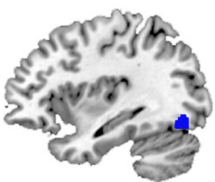

Cl1

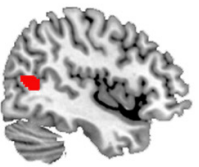

$\mathrm{Cl} 2$

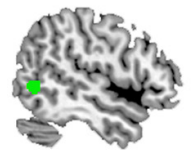

Cl3

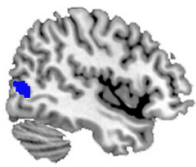

Cl4

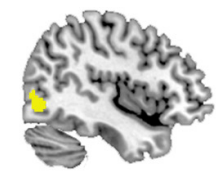

P(Activation|Domain)

Action.observation

Perception.vision shape

Interoception sexuality

Perception.vision motion

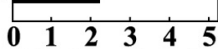

Likelihood ratio

Perception.vision shape Interoception sexuality Action.observation

Perception.vision motion Cognition.attention Cognition space

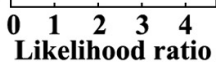

Perception.vision motion Perception.vision

Perception.vision shape Cognition.language semantics

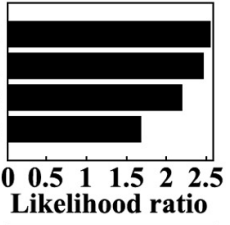

Perception.vision motion

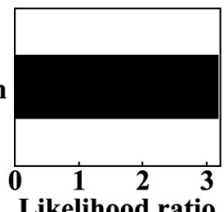

Action.observation

Perception.vision motion

Perception.vision shape
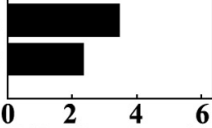

Likelihood ratio

Perception.vision motion Action.observation

Perception.vision

Cognition.soma

Cognition.space Interoception sexuality

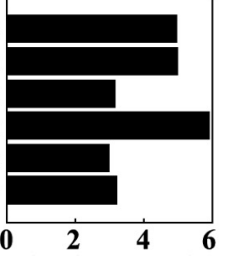

Likelihood ratio

Perception.vision shape Cognition.space Perception.vision motion Action.observation Perception.vision Cognition.soma Interoception sexuality

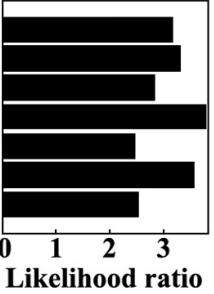

P(Activation|Paradigm)

Action observation Visual pursuit/tracking Film viewing Passive viewing Mental rotation

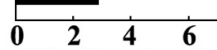

Likelihood ratio

Passive viewing

Action observation Mental rotation

Visual pursuit/tracking

Film viewing

Visual distractor/visual attention

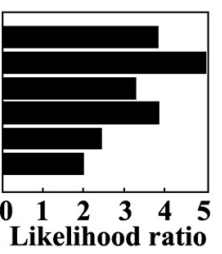

Visual pursuit/tracking

Naming(covert)

Visual distractor/visual attention

Visual distractor/visual attention
Passive viewing

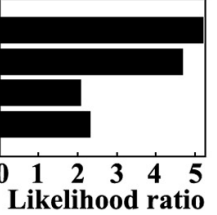

Face monitor/discrimination

Visual distractor/visual attention

Visuospatial attention

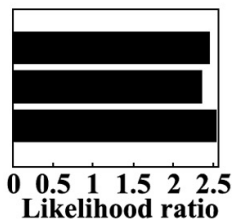

Action.observation Visual pursuit/tracking

Passive viewing

Affective pictures

Emotion induction

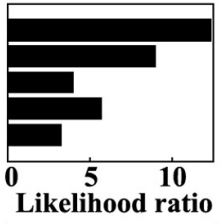

Visual pursuit/tracking

Passive viewing

Theory of mind

Naming(covert)

Film viewing

Action.observation

Mental rotation

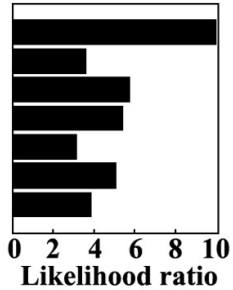

Passive viewing

Naming(covert)

Mental rotation

Action.observation

Visuospatial attention

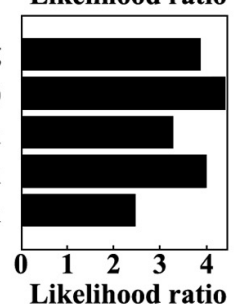

FIGURE 4 | Quantitative functional characterization of each subregion in the left (A) and right (B) MT was also performed. Significance was established using a binomial test $(p<0.05$ corrected for multiple comparisons using the FDR method) in the forward inference, whereas it was assessed by means of a Chi-squared test $(p<0.05$ corrected for multiple comparisons using Bonferroni's method) in the reverse inference approach.

As expected, all subregions of MT were mainly associated with vision motion. This result was consistent with previous studies that MT is the most known visual MT to detect and signal the presence and direction of visual motion (Lagae et al., 1994; Zeki, 2015). Moreover, most physiological studies showed that many of its cells rather than with that of its component parts are especially associated with the overall, global, direction of an object (Rust et al., 2006), strongly providing the structural fundament of its role in visual motion. Generally, the MT can not only receive information regarding visual motion from the extrastriate area, V6 along a dorsolateral visual stream, but also receive a direct input from $\mathrm{V} 1$ and the extrastriate areas of the 
occipital pole (Zeki et al., 1991). Moreover, evidence showed that neurons of MT are highly sensitive to the speed and direction of visual stimuli in motion (Albright, 1984). It has also been confirmed by several EEG and magnetoencephalographic (MEG) studies (Kawakami et al., 2002; Maruyama et al., 2002; Heinrich, 2007), which showed strong correlations between the latency and amplitude of the evoked response in the visual MT cortical area and the speed of the moving visual stimuli in the adults. Recently, a MEG study showed that the horizontal movement of the visual stimulus evoked changes in the strength of the theta-alpha (5$10 \mathrm{~Hz})$ and alpha-beta $(8-20 \mathrm{~Hz})$ oscillations in the visual MT area of all participants (VerMaas et al., 2019).

In addition to visual motion, we also identified distinct and specific functions associated with different subregions characterized by task-dependent functional connectivity mapping and forward/reverse inference. Among these, two findings draw our attention and worth to be emphasized: First, the left $\mathrm{Cl} 2$ was also involved in cognition attention based on the results of our functional characterization. Attentional mechanisms also form part of the repertoire of every visual area (VerMaas et al., 2019) but may be utilized differently in different visual areas (Maunsell and Cook, 2002). Several studies showed that the activity of MT can be affected by attention to visual motion (Buchel et al., 1998), because individuals usually attend to the task in the active state. In particular, studies reported modulation of responses in MT from parietal regions involved in selective attention and proposed that these regions modulate the effective connectivity from early visual cortex to the motion-sensitive area MT (Friston and Buchel, 2000). Moreover, our results also showed unique task-dependent connectivity between left $\mathrm{Cl} 2$ and bilateral IPS, which is the principal node in dorsal fronto-parietal attention network (Mayer et al., 2006; Connolly et al., 2016). In addition, greater attention-related activations in MT were identified in a task of following arrow cues (Callejas et al., 2014). Considering these information, the previously identified MT associated with dorsal fronto-parietal attention network might be confined to the left $\mathrm{Cl} 2$. Second, we also found that the left $\mathrm{Cl} 3$ is associated with language semantics. This result was functionally supported by the specific task-dependent connections between left $\mathrm{Cl} 3$ and classical language regions, such as IFG and SMG. These two regions have been widely and consistently reported to be associated with semantic processing (Rodd et al., 2015; Xu et al., 2016). Indeed, an fMRI study found that MT was activated significantly more for motion sentences than the other sentence types, suggesting that the neural substrates of linguistic semantics include early visual areas specifically related to the represented semantics (Saygin et al., 2010). Moreover, several behavioral studies also suggested that language can be interacted with low-level motion processing

\section{REFERENCES}

Abdollahi, R. O., Kolster, H., Glasser, M. F., Robinson, E. C., Coalson, T. S., Dierker, D., et al. (2014). Correspondences between retinotopic areas and myelin maps in human visual cortex. Neuroimage 99, 509-524. doi: 10.1016/j.neuroimage. 2014.06.042
(Meteyard et al., 2007, 2008). However, more evidence is still needed to confirm the role of left $\mathrm{Cl} 3$ in semantic processing using more direct approaches.

\section{CONCLUSION}

In conclusion, we identified two dorsal subregions ( $\mathrm{Cl1}$ and $\mathrm{Cl} 2)$ and one ventral subregion $(\mathrm{Cl} 3)$ of left $\mathrm{MT}$, as well as two dorsal-anterior subregions $(\mathrm{Cl} 1$ and $\mathrm{Cl} 2)$, one ventral-anterior subregion $(\mathrm{Cl} 3)$, and an additional posterior subregion $(\mathrm{Cl} 4)$ of right MT using coactivation-based parcellation. These subregions showed distinct and specific functions characterized by taskdependent functional connectivity mapping and forward/reverse inference in addition to vision motion. These results not only were in accordance with the previous findings of a hemispheric asymmetry of MT, but also strongly confirmed the existence of subregions in this region with distinct and specific functions. Furthermore, our results extend the special role of visual motion perception on this area and might facilitate future cognitive study. Although the MT area was parcellated into different functional subregions using coactivation-based parcellation, future studies with anatomical or resting-state functional connectivity-based parcellation are needed to further validate the current findings.

\section{DATA AVAILABILITY STATEMENT}

Publicly available datasets were analyzed in this study. This data can be found here: www.brainmap.org.

\section{AUTHOR CONTRIBUTIONS}

All authors listed have made a substantial, direct and intellectual contribution to the work, and approved it for publication.

\section{FUNDING}

This work was supported by the Applied Basic Research Programs of Sichuan Province (2019YJ0193), a Guangdong Key Basic Research Grant (2018B030332001), the Guangdong Pearl River Talents Plan (2016ZT06S220), the Shenzhen Science and Technology Research Program (Nos. JCYJ20170317155203481 and JCYJ20170412174037594), the Key R\&D Program of Guangdong Province (No. 2018B030339001), and the National Natural Science Foundation of China (61701078, 61671440, 31700964, and U1713215).

Albright, T. D. (1984). Direction and orientation selectivity of neurons in visual area MT of the macaque. J. Neurophysiol. 52, 11061130 .

Allman, J. M., and Kaas, J. H. (1971). A representation of the visual field in the caudal third of the middle tempral gyrus of the owl monkey (Aotus trivirgatus). Brain Res. 31, 85-105. 
Amano, K., Wandell, B. A., and Dumoulin, S. O. (2009). Visual field maps, population receptive field sizes, and visual field coverage in the human MT+ complex. J. Neurophysiol. 102, 2704-2718. doi: 10.1152/jn.00102.2009

Annese, J., Gazzaniga, M., and Toga, A. (2004). Localization of the human cortical visual area MT based on computer aided histological analysis. Cereb. Cortex. 15, 1044-1053.

Bridge, H., Clare, S., and Krug, K. (2014). Delineating extrastriate visual area MT(V5) using cortical myeloarchitecture. Neuroimage 93(Pt 2), 231-236. doi: 10.1016/j.neuroimage.2013.03.034

Buchel, C., Josephs, O., Rees, G., Turner, R., Frith, C. D., and Friston, K. J. (1998). The functional anatomy of attention to visual motion: a functional MRI study. Brain 121(Pt 7), 1281-1294.

Bzdok, D., Laird, A. R., Zilles, K., Fox, P. T., and Eickhoff, S. B. (2013). An investigation of the structural, connectional, and functional subspecialization in the human amygdala. Hum. Brain Mapp. 34, 3247-3266. doi: 10.1002/hbm. 22138

Callejas, A., Shulman, G. L., and Corbetta, M. (2014). Dorsal and ventral attention systems underlie social and symbolic cueing. J. Cogn. Neurosci. 26, 63-80. doi: 10.1162/jocn_a_00461

Cieslik, E. C., Zilles, K., Caspers, S., Roski, C., Kellermann, T. S., Jakobs, O., et al. (2013). Is there "one" DLPFC in cognitive action control? Evidence for heterogeneity from co-activation-based parcellation. Cereb. Cortex 23, 26772689. doi: $10.1093 /$ cercor/bhs 256

Clos, M., Amunts, K., Laird, A. R., Fox, P. T., and Eickhoff, S. B. (2013). Tackling the multifunctional nature of Broca's region meta-analytically: co-activation-based parcellation of area 44. Neuroimage 83, 174-188. doi: 10.1016/j.neuroimage. 2013.06.041

Connolly, J. D., Kentridge, R. W., and Cavina-Pratesi, C. (2016). Coding of attention across the human intraparietal sulcus. Exp. Brain Res. 234, 917-930. doi: 10.1007/s00221-015-4507-2

Dubner, R., and Zeki, S. M. (1971). Response properties and receptive fields of cells in an anatomically defined region of the superior temporal sulcus in the monkey. Brain Res. 35, 528-532.

Dumoulin, S. O., Bittar, R. G., Kabani, N. J., Baker, C. L. Jr., Le Goualher, G., Pike, G. B., et al. (2000). A new anatomical landmark for reliable identification of human area V5/MT: a quantitative analysis of sulcal patterning. Cereb. Cortex 10, 454-463.

Eickhoff, S. B., Bzdok, D., Laird, A. R., Kurth, F., and Fox, P. T. (2012). Activation likelihood estimation meta-analysis revisited. Neuroimage 59, 2349-2361. doi: 10.1016/j.neuroimage.2011.09.017

Eickhoff, S. B., Bzdok, D., Laird, A. R., Roski, C., Caspers, S., Zilles, K., et al. (2011). Co-activation patterns distinguish cortical modules, their connectivity and functional differentiation. Neuroimage 57, 938-949. doi: 10.1016/j.neuroimage. 2011.05.021

Eickhoff, S. B., Jbabdi, S., Caspers, S., Laird, A. R., Fox, P. T., Zilles, K., et al. (2010). Anatomical and functional connectivity of cytoarchitectonic areas within the human parietal operculum. J. Neurosci. 30, 6409-6421.

Eickhoff, S. B., Laird, A. R., Grefkes, C., Wang, L. E., Zilles, K., and Fox, P. T. (2009). Coordinate-based activation likelihood estimation meta-analysis of neuroimaging data: a random-effects approach based on empirical estimates of spatial uncertainty. Hum. Brain Mapp. 30, 2907-2926. doi: 10.1002/hbm.20718

Ferri, S., Kolster, H., Jastorff, J., and Orban, G. A. (2013). The overlap of the EBA and the MT/V5 cluster. Neuroimage 66, 412-425. doi: 10.1016/j.neuroimage. 2012.10.060

Friston, K. J., and Buchel, C. (2000). Attentional modulation of effective connectivity from V2 to V5/MT in humans. Proc. Natl. Acad. Sci. U.S.A. 97, 7591-7596.

Glasser, M. F., and van Essen, D. C. (2011). Mapping human cortical areas in vivo based on myelin content as revealed by T1-and T2-weighted MRI. J. Neurosci. 31, 11597-11616. doi: 10.1523/JNEUROSCI.2180-11.2011

Heinrich, S. P. (2007). A primer on motion visual evoked potentials. Doc. Ophthalmol. 114, 83-105.

Howard, R., Bullmore, E., Brammer, M., Williams, S., Mellers, J., Woodruff, P., et al. (1995). Activation of area V5 by visual perception of motion demonstrated with echoplanar MR imaging. Magn. Reson. Imaging 13, 907-909.

Huk, A. C., Dougherty, R. F., and Heeger, D. J. (2002). Retinotopy and functional subdivision of human areas MT and MST. Neuroscience 22, 7195-7205.
Kahnt, T., Chang, L. J., Park, S. Q., Heinzle, J., and Haynes, J. D. (2012). Connectivity-based parcellation of the human orbitofrontal cortex. J. Neurosci. 32, 6240-6250.

Kawakami, O., Kaneoke, Y., Maruyama, K., Kakigi, R., Okada, T., Sadato, N., et al. (2002). Visual detection of motion speed in humans: spatiotemporal analysis by fMRI and MEG. Hum. Brain Mapp. 16, 104-118.

Kolster, H., Peeters, R., and Orban, G. A. (2010). The retinotopic organization of the human middle temporal area MT/V5 and its cortical neighbors. J. Neurosci. 30, 9801-9820. doi: 10.1523/JNEUROSCI.2069-10.2010

Lagae, L., Maes, H., Raiguel, S., Xiao, D. K., and Orban, G. A. (1994). Responses of macaque STS neurons to optic flow components: a comparison of areas MT and MST. J. Neurophysiol. 71, 1597-1626.

Laird, A. R., Eickhoff, S. B., Fox, P. M., Uecker, A. M., Ray, K. L., Saenz, J. J. Jr., et al. (2011). The BrainMap strategy for standardization, sharing, and meta-analysis of neuroimaging data. BMC Res. Notes 4:349. doi: 10.1186/1756-0500-4-349

Laird, A. R., Eickhoff, S. B., Kurth, F., Fox, P. M., Uecker, A. M., Turner, J. A., et al. (2009). ALE meta-analysis workflows via the BrainMap database: progress towards a probabilistic functional brain atlas. Front. Neuroinform. 3:23. doi: 10.3389/neuro.11.023.2009

Laird, A. R., Eickhoff, S. B., Rottschy, C., Bzdok, D., Ray, K. L., and Fox, P. T. (2013). Networks of task co-activations. Neuroimage 80, 505-514. doi: 10.1016/ j.neuroimage.2013.04.073

Maruyama, K., Kaneoke, Y., Watanabe, K., and Kakigi, R. (2002). Human cortical responses to coherent and incoherent motion as measured by magnetoencephalography. Neurosci. Res. 44, 195-205.

Maunsell, J. H., and Cook, E. P. (2002). The role of attention in visual processing. Philos. Trans. R. Soc. Lond. B Biol. Sci. 357, 1063-1072.

Mayer, A. R., Harrington, D., Adair, J. C., and Lee, R. (2006). The neural networks underlying endogenous auditory covert orienting and reorienting. Neuroimage 30, 938-949.

Meteyard, L., Bahrami, B., and Vigliocco, G. (2007). Motion detection and motion verbs: language affects low-level visual perception. Psychol. Sci. 18, 1007-1013.

Meteyard, L., Zokaei, N., Bahrami, B., and Vigliocco, G. (2008). Visual motion interferes with lexical decision on motion words. Curr. Biol. 18, R732-R733.

Morrone, M., Tosetti, M., Montanaro, D., Fiorentini, A., Cioni, G., and Burr, D. (2000). A cortical area that responds specifically to optic flow, revealed by fMRI. Nat. Neurosci. 3:1322.

Ohlendorf, S., Sprenger, A., Speck, O., Haller, S., and Kimmig, H. (2008). Optic flow stimuli in and near the visual field centre: a group FMRI study of motion sensitive regions. PLoS One 3:e4043. doi: 10.1371/journal.pone.0004043

Poirier, C., Collignon, O., Scheiber, C., Renier, L., Vanlierde, A., Tranduy, D., et al. (2006). Auditory motion perception activates visual motion areas in early blind subjects. Neuroimage 31, 279-285.

Ricciardi, E., Vanello, N., Sani, L., Gentili, C., Scilingo, E. P., Landini, L., et al. (2007). The effect of visual experience on the development of functional architecture in hMT+. Cereb. Cortex 17, 2933-2939.

Robinson, J. L., Laird, A. R., Glahn, D. C., Lovallo, W. R., and Fox, P. T. (2010). Metaanalytic connectivity modeling: delineating the functional connectivity of the human amygdala. Hum. Brain Mapp. 31, 173-184. doi: 10.1002/hbm.20854

Rodd, J. M., Vitello, S., Woollams, A. M., and Adank, P. (2015). Localising semantic and syntactic processing in spoken and written language comprehension: an activation likelihood estimation meta-analysis. Brain Lang. 141, 89-102. doi: 10.1016/j.bandl.2014.11.012

Rottschy, C., Caspers, S., Roski, C., Reetz, K., Dogan, I., Schulz, J. B., et al. (2013). Differentiated parietal connectivity of frontal regions for "what" and "where" memory. Brain Structure Funct. 218, 1551-1567. doi: 10.1007/s00429-0120476-4

Rust, N. C., Mante, V., Simoncelli, E. P., and Movshon, J. A. (2006). How MT cells analyze the motion of visual patterns. Nat. Neurosci. 9, 1421-1431.

Sanchez-Panchuelo, R. M., Francis, S. T., Schluppeck, D., and Bowtell, R. W. (2012). Correspondence of human visual areas identified using functional and anatomical MRI in vivo at 7 T. J. Magn. Reson. Imaging 35, 287-299. doi: 10.1002/jmri.22822

Saygin, A. P., McCullough, S., Alac, M., and Emmorey, K. (2010). Modulation of BOLD response in motion-sensitive lateral temporal cortex by real and fictive motion sentences. J. Cogn. Neurosci. 22, 2480-2490. doi: 10.1162/jocn.2009. 21388 
Sereno, M. I., Lutti, A., Weiskopf, N., and Dick, F. (2013). Mapping the human cortical surface by combining quantitative $\mathrm{T}(1)$ with retinotopy. Cereb. Cortex 23, 2261-2268. doi: 10.1093/cercor/bhs213

Smith, A. T., Wall, M. B., Williams, A. L., and Singh, K. D. (2006). Sensitivity to optic flow in human cortical areas MT and MST. Eur. J. Neurosci. 23, 561-569.

Tootell, R. B., Reppas, J. B., Dale, A. M., Look, R. B., Sereno, M. I., Malach, R., et al. (1995). Visual motion aftereffect in human cortical area MT revealed by functional magnetic resonance imaging. Nature 375, 139-141.

Turkeltaub, P. E., Eickhoff, S. B., Laird, A. R., Fox, M., Wiener, M., and Fox, P. (2012). Minimizing within-experiment and within-group effects in activation likelihood estimation meta-analyses. Hum. Brain Mapp. 33, 1-13. doi: 10.1002/ hbm.21186

VerMaas, J. R., Gehringer, J. E., Wilson, T. W., and Kurz, M. J. (2019). Children with cerebral palsy display altered neural oscillations within the visual MT/V5 cortices. Neuroimage Clin. 23:101876. doi: 10.1016/j.nicl.2019.101876

Wang, J., Becker, B., Wang, L., Li, H., Zhao, X., and Jiang, T. (2019). Corresponding anatomical and coactivation architecture of the human precuneus showing similar connectivity patterns with macaques. Neuroimage 200, 562-574. doi: 10.1016/j.neuroimage.2019.07.001

Wang, J., Fan, L., Wang, Y., Xu, W., Jiang, T., Fox, P. T., et al. (2015a). Determination of the posterior boundary of Wernicke's area based on multimodal connectivity profiles. Hum. Brain Mapp. 36, 1908-1924. doi: 10. 1002/hbm. 22745

Wang, J., Fan, L., Zhang, Y., Liu, Y., Jiang, D., Zhang, Y., et al. (2012). Tractography-based parcellation of the human left inferior parietal lobule. Neuroimage 63, 641-652. doi: 10.1016/j.neuroimage.2012.07.045

Wang, J., Xie, S., Guo, X., Becker, B., Fox, P. T., Eickhoff, S. B., et al. (2017). Correspondent functional topography of the human left inferior parietal lobule at rest and under task revealed using resting-state fMRI and coactivation based parcellation. Hum. Brain Mapp. 38, 1659-1675. doi: 10.1002/hbm.23488

Wang, J., Yang, Y., Fan, L. Z., Xu, J. P., Li, C. H., Liu, Y., et al. (2015b). Convergent functional architecture of the superior parietal lobule unraveled with multimodal neuroimaging approaches. Hum. Brain Mapp. 36, 238-257. doi: $10.1002 / \mathrm{hbm} .22626$

Wang, J., Zhang, J., Rong, M., Wei, X., Zheng, D., Fox, P. T., et al. (2016). Functional topography of the right inferior parietal lobule structured by anatomical connectivity profiles. Hum. Brain Mapp. 37, 4316-4332. doi: 10. 1002/hbm. 23311

Watkins, K. E., Shakespeare, T. J., O’Donoghue, M. C., Alexander, I., Ragge, N., Cowey, A., et al. (2013). Early auditory processing in area V5/MT+ of the congenitally blind brain. J. Neurosci. 33, 18242-18246. doi: 10.1523/ JNEUROSCI.2546-13.2013
Watson, J. D., Myers, R., Frackowiak, R. S., Hajnal, J. V., Woods, R. P., Mazziotta, J. C., et al. (1993). Area V5 of the human brain: evidence from a combined study using positron emission tomography and magnetic resonance imaging. Cereb. Cortex 3, 79-94.

Xu, J., Lyu, H., Li, T., Xu, Z., Fu, X., Jia, F., et al. (2019a). Delineating functional segregations of the human middle temporal gyrus with resting-state functional connectivity and coactivation patterns. Hum. Brain Mapp. 40, 5159-5171. doi: 10.1002/hbm. 24763

Xu, J., Wang, C., Xu, Z., Li, T., Chen, F., Chen, K., et al. (2019b). Specific functional connectivity patterns of middle temporal gyrus subregions in children and adults with autism spectrum disorder. Autism Res. 13, 410-422. doi: 10.1002/ aur.2239

Xu, J., Wang, J., Fan, L., Li, H., Zhang, W., Hu, Q., et al. (2015). Tractographybased parcellation of the human middle temporal gyrus. Sci. Rep. 5:18883. doi: 10.1038/srep18883

Xu, Y., Lin, Q., Han, Z., He, Y., and Bi, Y. (2016). Intrinsic functional network architecture of human semantic processing: modules and hubs. Neuroimage 132, 542-555. doi: 10.1016/j.neuroimage.2016.03.004

Zeki, S. (2015). Area V5-a microcosm of the visual brain. Front. Integr. Neurosci. 9:21. doi: 10.3389/fnint.2015.00021

Zeki, S., Watson, J. D., Lueck, C. J., Friston, K. J., Kennard, C., and Frackowiak, R. S. (1991). A direct demonstration of functional specialization in human visual cortex. J. Neurosci. 11, 641-649.

Zeki, S. M. (1974). Functional organization of a visual area in the posterior bank of the superior temporal sulcus of the rhesus monkey. J. Physiol. 236, 549-573.

Zhang, Y., Han, B., Verhaeghen, P., and Nilsson, L. G. (2007). Executive functioning in older adults with mild cognitive impairment: MCI has effects on planning, but not on inhibition. Neuropsychol. Dev. Cogn. B Aging Neuropsychol. Cogn. 14, 557-570.

Conflict of Interest: The authors declare that the research was conducted in the absence of any commercial or financial relationships that could be construed as a potential conflict of interest.

Copyright (c) 2020 Gao, Zeng, Dai, Yang, Yu, Chen, Hu, Xu, Cheng and Wang. This is an open-access article distributed under the terms of the Creative Commons Attribution License (CC BY). The use, distribution or reproduction in other forums is permitted, provided the original author(s) and the copyright owner(s) are credited and that the original publication in this journal is cited, in accordance with accepted academic practice. No use, distribution or reproduction is permitted which does not comply with these terms. 